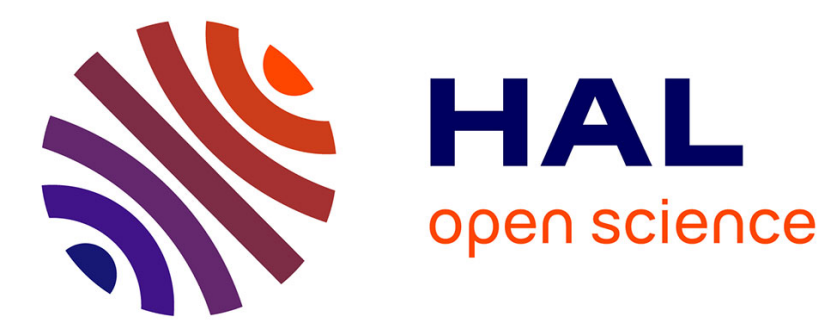

\title{
Constraining the nuclear pairing gap with pairing vibrations
}

E. Khan, Marcella Grasso, Jerome Margueron

\section{To cite this version:}

E. Khan, Marcella Grasso, Jerome Margueron. Constraining the nuclear pairing gap with pairing vibrations. Physical Review C, 2009, 80, pp.044328. 10.1103/PhysRevC.80.044328 . in2p3-00387983

\section{HAL Id: in2p3-00387983 \\ https://hal.in2p3.fr/in2p3-00387983}

Submitted on 26 May 2009

HAL is a multi-disciplinary open access archive for the deposit and dissemination of scientific research documents, whether they are published or not. The documents may come from teaching and research institutions in France or abroad, or from public or private research centers.
L'archive ouverte pluridisciplinaire HAL, est destinée au dépôt et à la diffusion de documents scientifiques de niveau recherche, publiés ou non, émanant des établissements d'enseignement et de recherche français ou étrangers, des laboratoires publics ou privés. 


\title{
Constraining the nuclear pairing gap with pairing vibrations
}

\author{
E. Khan, ${ }^{1}$ M. Grasso, ${ }^{1}$ and J. Margueron ${ }^{1}$ \\ ${ }^{1}$ Institut de Physique Nucléaire, Université Paris-Sud, IN2P3-CNRS, F-91406 Orsay Cedex, France
}

\begin{abstract}
Pairing interactions with various density dependencies (surface/volume mixing) are constrained with the two-neutron separation energy in the Tin isotopic chain. The response associated with pairing vibrations in very neutron-rich nuclei is sensitive to the density dependence of the pairing interaction. Using the same pairing interaction in nuclear matter and in Tin nuclei, the range of densities where the LDA is valid in the pairing channel is also studied.
\end{abstract}

PACS numbers: 21.60.Jz,21.65.Cd,25.40.Hs,25.60.Je

\section{INTRODUCTION}

Studies on pairing effects in both nuclear matter and finite nuclei have known intensified interests in the recent years [1]. There are two main approaches for pairing, depending whether the mean field is based on Gogny finite range interaction or on Skyrme interaction. In the first approach, a similar functional is used in both the particle-hole channel and the pairing channel, although interactions are not exactly the same due to the density dependence of the pairing interaction. In the Skyrme approach, the functionals are meant to be different in the two channels, as witnessed for instance by their density dependence. The use of a different interaction in the particle-hole channel and in the pairing channel has been justified a decade ago [2]; this is for instance the case of employing the Skyrme interaction in the particle-hole channel and a zero range density dependent interaction in the pairing channel. We shall focus on the Skyrme approach: in this case the pairing density functional is difficult to constrain and it has not been possible to derive an universal pairing interaction during past decades, using for instance the odd-even mass staggering on finite nuclei (see e.g. [3, 4). This may indicate the need for another approach, using additional constrains: should the pairing density functional be extended, and are there additional relevant observables to constrain it ?

Nuclear matter could help in constraining the pairing functional. This requires however to bridge nuclei and nuclear matter through LDA in the pairing channel: its condition of validity should be more systematically analysed. It has been recently shown that the two paired neutrons are spatially localised in low density medium which corresponds to the surface of the nucleus [0]. The same conclusion is drawn by analysing the di-neutron configuration in the excited states [6, 7], and also performing calculations in low density matter in [8, 9], mainly renewing the possibility to link in some cases the nuclear matter and nuclei in the pairing channel through the LDA.

Concomitantly the pairing functional has been extended in order to study the condensation of the Cooper pairs (BEC-BCS crossover) in both symmetric and neutron matter. In nuclear matter the medium polarization increases the pairing gap at low densities in symmetric matter, whereas it reduces the gap in neutron matter, in- dicating an isospin dependence of the pairing functional [10]. The application to finite nuclei of extended pairing density functional have shown the relevance of the LDA in the pairing channel [11].

The pairing functional studies may thus enter in a new era, renewing the method to design the pairing interaction: i) using an isospin dependence of the pairing interaction ii) using eventually the nuclear matter as an additional constrain for the pairing interaction iii) looking for additional observables in nuclei than the odd-even mass staggering to constrain the pairing interaction. Point i) has been investigated in 10, 11]. Point ii) requires the validity of the LDA in the pairing channel and shall be addressed in the present work.

In the case of point iii) an interesting observable is pairing vibrations, measured through two-particle transfer. It is well known that the transfer cross section crucially depends on the pairing interaction at work in the transferred paired [12, 13. However in the 70-80's the form factor of the transition has never been calculated fully microscopically. The first microscopic calculations has been performed only recently [14], allowing for a strong link between the pairing interaction and pairing vibrations. Several calculations followed [6, 15], showing the renewed interest for such studies.

It is therefore meaningful to use pairing vibrations as a complementary observable to the masses, in order to constrain the pairing interaction, and study the implications to the nuclear matter. More generally, the isospin extension of the pairing interaction should be accompanied with additional constraints. One purpose of this work is to evaluate if pairing vibrations could play this role (Section III).

The method is to analyse the sensitivity of pairing vibrations to various pairing interactions which provide the same two-neutron separation energy in Tin isotopes, and evaluate the consequences on the pairing gap in symmetric and neutron matter. On this purpose it is necessary to determine the range of density where the LDA is valid in the pairing channel (Section II). 
TABLE I: Values of $\eta$ and $V_{0}$ of the pairing interaction.

\begin{tabular}{cc}
\hline \hline$\eta$ & $V_{0}\left(\mathrm{MeV} \mathrm{fm}^{-1}\right)$ \\
\hline 0.35 & -285 \\
0.65 & -390 \\
1 & -670 \\
\hline \hline
\end{tabular}

\section{VALIDITY OF THE LDA IN THE PAIRING CHANNEL}

After many years of study, there is still no unambiguous universal pairing functional ranging on the whole nuclear chart, and current efforts are aiming in that direction. The problem may be due to the method used to constrain it, namely comparing the pairing gap with odd-even mass differences, or evaluating the separation energies along a given isotopic chain. It therefore may be useful to consider a more general context: the evaluation of several pairing interactions constrained by odd-even mass difference, on nuclear matter on one side, and on additional observables on the other side, should shed a renewed light on the problem. To achieve this goal it is first necessary to determine the range of validity of the LDA in the pairing channel.

\section{A. Method to determine the functional}

The method is the following: we first consider surface and various mixed paring interactions. The parameters are determined so as to describe the two neutron separation energy. Then pairing vibrations are used in order to disentangle between the various pairing interactions (Section III). We choose ${ }^{124} \mathrm{Sn}$ and ${ }^{136} \mathrm{Sn}$ nuclei: these are spherical nuclei where pairing vibrations are likely to occur [13]. One is stable and the second has a large neutron excess.

The microscopic calculations for the ground state are based on the Hartree-Fock-Bogoliubov (HFB) model. The Skyrme interaction SLy4 [16] is chosen for the particle-hole channel of the HFB equations. The adopted pairing interaction is the usual zero-range densitydependent interaction

$$
V_{\text {pair }}=V_{0}\left[1-\eta\left(\frac{\rho(r)}{\rho_{0}}\right)^{\alpha}\right] \delta\left(\mathbf{r}_{1}-\mathbf{r}_{2}\right)
$$

where $\eta$ provides the surface/volume character of the interaction. We set $\alpha=1$ and $\rho_{0}=0.16 \mathrm{fm}^{-3}$. The numerical cutoff for the microscopic calculations is given by $E_{\max }=60 \mathrm{MeV}$ (in quasiparticle energies) and $j_{\max }=$ $15 / 2$. For each value of $\eta, V_{0}$ is chosen to fit the known experimental two-neutron separation energies for Sn isotopes. Surface and mixed interactions have been considered in this work and the used values of $\left(\eta, V_{0}\right)$ are listed in Table I.

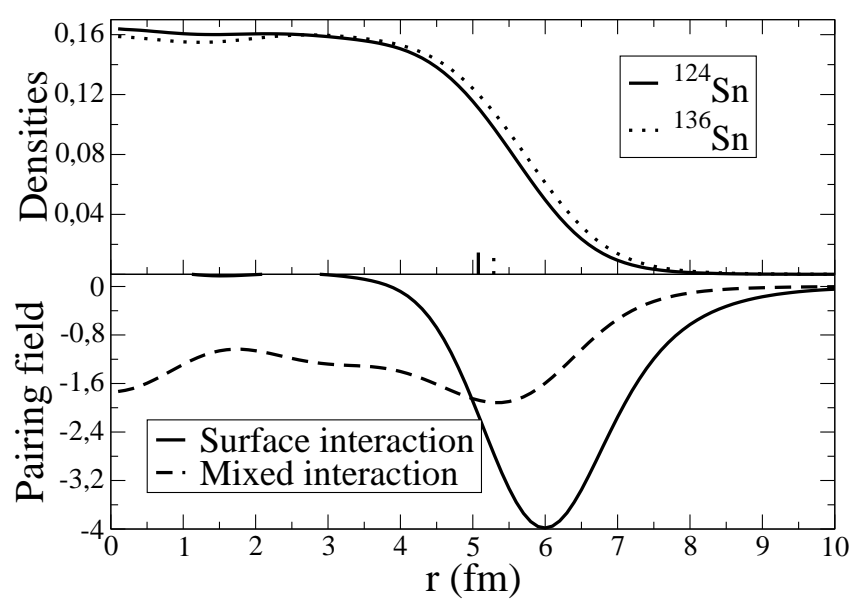

FIG. 1: Upper panel: Matter densities calculated with the HFB model for ${ }^{124} \mathrm{Sn}$ and ${ }^{136} \mathrm{Sn}$. The vertical lines indicate the radius corresponding the density at which all the pairing interactions converge in uniform matter (see text). Lower panel: pairing field of ${ }^{124} \mathrm{Sn}$ calculated with a surface $\eta=1$ (top) and a mixed $\eta=0.35$ (bottom) pairing interaction.

As an illustration to visualize the features of the calculated pairing effects, we display in Fig. 1 the neutron pairing field for ${ }^{124} \mathrm{Sn}$ corresponding to the surface $\eta=1$ and the mixed $\eta=0.35$ interactions.

\section{B. Pairing gap in uniform matter}

The relation between the pairing gap in uniform matter at a given density and the pairing field at a given radius in nuclei has been explored in Ref. 11]. It has been found that in the case of mixed interactions, the LDA is in good agreement with the full microscopic HFB calculation (differences less than $15 \%$ on the pairing field). This might be related to the extension of the Cooper pair which is getting smaller at the surface of nuclei (about $2 \mathrm{fm}$ ) compared to that in the interior (about 5-6 fm) [5]. Close to the surface, pairing properties shall not be very different from that of a uniform piece of matter at the same density. It is then interesting to explore the low density properties of the different pairing interactions listed in Table I.

Fig. 2 displays the pairing gap in uniform matter for various pairing interactions. It is observed that the different interactions leads to very different pairing gap at low density while around saturation density, there is a density $\left(\rho=0.11 \mathrm{fm}^{-3}\right)$ at which the pairing gap and pairing strength coincide for the three pairing interactions.

From Fig. 2, two conclusions can be drawn: i) the twoneutron separation energy used to adjust the parameters of the pairing interaction is an observable which provides a strong constrain on the pairing gap localized at the surface of the nuclei: the pairing gap in nuclear matter has been constrained for $\rho=0.11 \mathrm{fm}^{-3}$, which corresponds to 


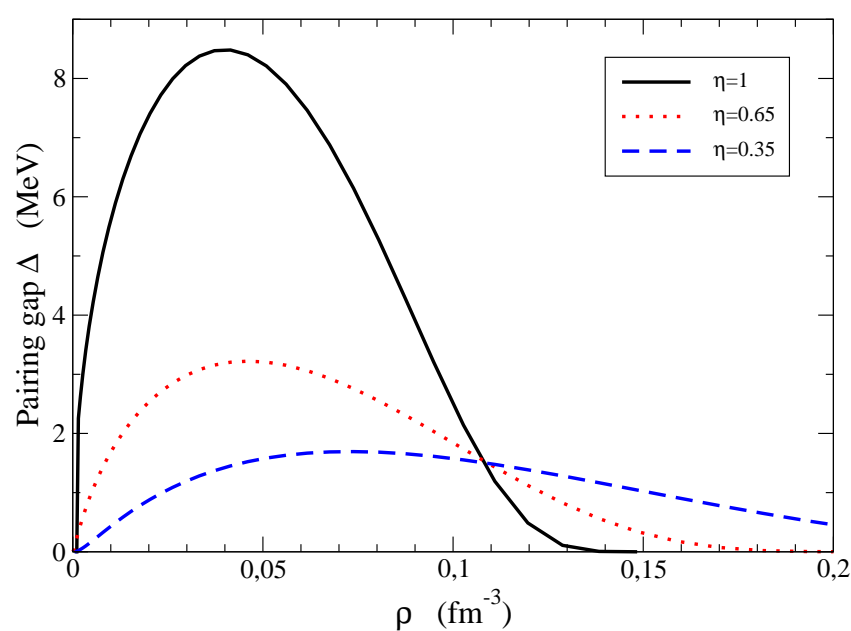

FIG. 2: Pairing gap versus the density for uniform matter for different pairing interactions.

$\mathrm{R} \simeq 5 \mathrm{fm}$ in Tin nuclei, as showed by the ${ }^{124} \mathrm{Sn}$ densities displayed on Fig. 1 1 ii) to better constrain the value of the parameter $\eta$, one shall find another observable sensitive to the pairing strength at low density (large radius, experimentally easier to probe). Indeed, in the very external part of the nuclei the pairing strength is very different from one interaction to another. The pure surface pairing interaction predict a pairing gap as high as $8 \mathrm{MeV}$ at low density while the various mixed pairing interaction are grouped below $3 \mathrm{MeV}$ (see Fig. 2).

Therefore, one might expect that properties of collective modes sensitive to the external part of the nuclei could be changed by the properties of the pairing interaction at low density. Pair transferred reaction mechanisms like $(\mathrm{p}, \mathrm{t})$ or $\left(\alpha,{ }^{6} \mathrm{He}\right)$ which is very surface peaked shall also help in extracting the value of the pairing gap in the external part of the nuclei or equivalently at low density.

\section{Validity of the LDA}

One aim of this work is to investigate the mapping of the pairing gap between nuclei and nuclear matter, implying the following relation:

$$
\Delta_{\infty}(\rho)=\Delta_{H F B}(\rho(r)=\rho)
$$

Eq. (2) corresponds to the LDA in the pairing channel. However, it should be noted that in practice, the LDA might still be valid even if Eq. (2) is not verified for very low densities $\left(\rho \leq 0.1 \mathrm{fm}^{-3}\right)$ : the major role is played by densities around the saturation density, where nucleus properties are the most important.

Fig. 3 displays the gap calculated with the HFB approach as a function of the nuclei density in ${ }^{124} \mathrm{Sn}$. Nuclear matter gap is surimposed, where the neutron-proton

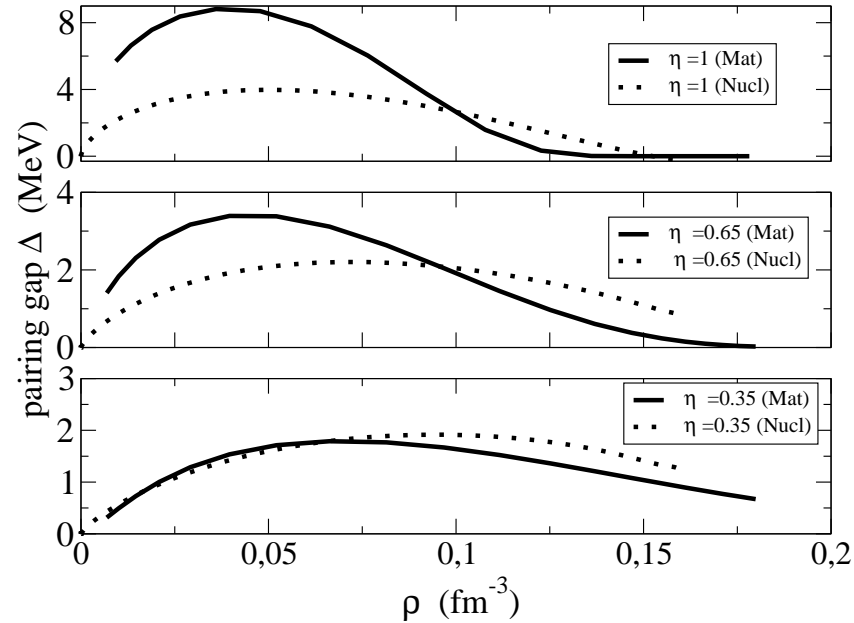

FIG. 3: Pairing gap versus the density for nuclear matter and for ${ }^{124} \mathrm{Sn}$ (see text)

asymmetry are taken as the one of ${ }^{124} \mathrm{Sn}$. One can see that the LDA is not valid for a pure surface pairing, but becomes valid in the $\eta=0.35$ mixed case, for densities lower than $0.1 \mathrm{fm}^{-3}$. Therefore, in this case, the low density part of the nuclear matter gap could be constrained by nuclei calculations of the gap: a valid case of the LDA in the pairing channel is showed here quantitatively for the first time. This range of density corresponds to a radius larger than about $5.1 \mathrm{fm}$ in ${ }^{124} \mathrm{Sn}$ and $5.2 \mathrm{fm}$ in ${ }^{136} \mathrm{Sn}$ localised at the surface of the nuclei, as can be seen on Fig. 1. In the cases of the other pairing interactions, the LDA underestimates the pairing gap at low density and overestimate it at high density. However, it should be noted that the relative difference of the gaps between the various pairing interactions are respected.

As noted above, to assess in practice the validity of the LDA, the density range around the saturation density should be investigated, where nuclei bulk properties are at work. Therefore a large discrepancy at very low density $\left(\rho \leq 0.1 \mathrm{fm}^{-3}\right)$, as observed in the case of the surface pairing interaction in Fig . 3, does not preclude on the validity of the LDA. More generally it can be stated that the less important the surface contribution to the pairing interaction, the better the LDA.

\section{PAIRING VIBRATIONS}

As stated above, it may be useful to consider an additional observable than the separation energy, in order to constrain the pairing interaction, namely its density dependence. There are only few observables which could be relevant to constrain pairing effects. It has been shown that the first $2^{+}$state in nuclei is sensitive to the pairing interaction [17]: both its position and strength depend of the pairing interaction. However this is mainly related to the pairing gap value, which is the same observable 
extracted from odd-even mass excess. It should be noted than none of these two observables (the first $2^{+}$state and the odd-even mass staggering) can be directly linked to predictions. On one side there is the difficulty to modelize excited states. On the other side, the difficulty is to describe odd nuclei.

Pairing vibrations may be more adequate observable. They can be probed for instance with two neutron transfer in nuclei close to shell closure. We refer to 12, 13] for details on pairing vibrations. Basically, these modes corresponds to the (collective) filling of subshells, in transition from an $\mathrm{A}$ to $\mathrm{A}+2$ nuclei.

With pairing vibrations, pairing effects are probed by 3 ways. The first one is the magnitude of the pairing gap $\Delta$ (average of the pairing field): a large pairing gap implies strength at larger energies, following the formula $\mathrm{E}^{2} \simeq(\epsilon-\lambda)^{2}+\Delta^{2}$. This component is also present in the first $2^{+}$state in the ph response as well as in the odd-even mass staggering. But in the case of the pairing vibrations, there are two additional contributions: first, the transition densities generating the strength are the pairing one, which means that the unperturbed response as well as the perturbed response are sensitive to the impact of the pairing on the wave functions. Finally, the residual interaction, generating the QRPA response, is also sensitive to pairing.

Pairing vibrations are therefore expected to be very sensitive to the pairing interaction. On the other hand, it may also be difficult to disentangle between the three above mentioned effects. However, the first one can be evaluated using the energies of the unperturbed response, the second one by studying the pairing transition densities, and the last one by comparing the unperturbed and the QRPA responses. It should be noted that a related study will also be performed in 18 .

\section{A. Method: QRPA in the pp channel}

The method is described in 14, 17. Namely the QRPA equations are solved in coordinate space, using the green function formalism. The variation of the generalized density $\mathcal{R}^{\prime}$ is expressed in term of 3 quantities, namely $\rho^{\prime}, \kappa^{\prime}$ and $\bar{\kappa}^{\prime}$, which are written as a column vector:

$$
\rho^{\prime}=\left(\begin{array}{c}
\rho^{\prime} \\
\kappa^{\prime} \\
\bar{\kappa}^{\prime}
\end{array}\right),
$$

where $\rho_{i j}^{\prime}=\left\langle 0\left|c_{j}^{\dagger} c_{i}\right|^{\prime}\right\rangle$ is the variation of the particle density, $\kappa_{i j}^{\prime}=\left\langle 0\left|c_{j} c_{i}\right|^{\prime}\right\rangle$ and $\bar{\kappa}_{i j}^{\prime}=\left\langle 0\left|c_{j}^{\dagger} c_{i}^{\dagger}\right|^{\prime}\right\rangle$ are the fluctuations of the pairing tensor associated to the pairing vibrations and $\left.\left.\right|^{\prime}\right\rangle$ denotes the change of the ground state wavefunction $\mid 0>$ due to the external field. In contrast with the RPA where one needs to know only the change of the ph density $\left(\rho^{\prime}\right)$, the variation of the three quantities (3) have to be calculated in the QRPA. In the three dimensional space introduced in Eq. (3), the first dimension represents the particle-hole (ph) subspace, the second the particle-particle (pp) one, and the third the hole-hole (hh) one. The response matrix has therefore 9 coupled elements in QRPA, compared to one in the RPA formalism.

The variation of the HFB Hamiltonian is given by:

$$
H^{\prime}=\mathbf{V} \rho^{\prime},
$$

where $\mathbf{V}$ is the matrix of the residual interaction expressed in terms of the second derivatives of the HFB energy functional, namely:

$$
\mathbf{V}^{\alpha \beta}\left(\mathbf{r} \sigma, \mathbf{r}^{\prime} \sigma^{\prime}\right)=\frac{\partial^{2} \mathcal{E}}{\partial \rho_{\beta}\left(\mathbf{r}^{\prime} \sigma^{\prime}\right) \partial \rho_{\bar{\alpha}}(\mathbf{r} \sigma)}, \quad \alpha, \beta=1,2,3 .
$$

In the above equation the notation $\bar{\alpha}$ means that whenever $\alpha$ is 2 or 3 then $\bar{\alpha}$ is 3 or 2 .

The QRPA Green's function G can be used for calculating the strength function associated with the twoparticle transfer from the ground state of a nucleus with A nucleons to the excited states of a nucleus with $\mathrm{A}+2$ nucleons. This strength function is :

$$
S(\omega)=-\frac{1}{\pi} \operatorname{Im} \int F^{*}(\mathbf{r}) \mathbf{G}^{22}\left(\mathbf{r}, \mathbf{r}^{\prime} ; \omega\right) F\left(\mathbf{r}^{\prime}\right) d \mathbf{r} d \mathbf{r}^{\prime}
$$

where $\mathbf{G}$ denotes the (pp,pp) component of the Green's function and $\mathrm{F}$ in the external perturbating field associated with the addition of two particles.

In the QRPA calculations the full HFB quasiparticle spectrum up to $60 \mathrm{MeV}$ is included. These states are used to construct the unperturbed Green's function $\mathbf{G}_{\mathbf{0}}$. The residual interaction is derived from the two-body force used in HFB according to Eq. (5). The contribution given by the velocity-dependent terms of the Skyrme force to the residual interaction is calculated in the Landau-Migdal approximation, which is shown to be accurate 19]. The strength function for the two-neutron transfer is calculated using Eq. (6). The unperturbed Green's function is calculated with an averaging interval equal to $0.15 \mathrm{MeV}$. All details can be found in Ref. [14.

The response function is calculated for the pp channel. All the calculations are performed in a box of size 22.5 $\mathrm{fm}$. It should be noted that exact continuum treatment is much heavier, especially for nuclei such as Sn isotopes. Moreover the aim is not to study the impact of the continuum treatment (see 14] for such a study). Finally the Sn isotopes under study are far from the drip line, and continuum effects are expected to play a negligible role.

\section{B. Unperturbed response results}

The HFB solutions are used in the quasiparticle random-phase approximation (QRPA) scheme to analyze 
self-consistently the excitations modes associated to the pair transfer reactions. Since we study here two-neutron transfers, we focus on the neutron HFB quasiparticle states that are used to construct the elementary configurations of the excited modes. We work with positiveenergy quasiparticle states. Once calculated the quasiparticle spectrum, it is possible to deduce some properties of the unperturbed response function.

The quasiparticle states with energy less than $6 \mathrm{MeV}$ and an occupation probability $\leq 80 \%$ are presented in Tables II and III for ${ }^{124} \mathrm{Sn}$ and ${ }^{136} \mathrm{Sn}$, respectively. Let us discuss the two cases $\eta=0.35$ and $\eta=1$ (for $\eta=0.65$, results are similar to those obtained with $\eta=0.35)$. For ${ }^{124} \mathrm{Sn}$, in the case of a mixed pairing interaction, $\eta=0.35$, all the quasiparticle states with energy lower than $5 \mathrm{MeV}$ are totally occupied with the exception of a $h_{11 / 2}$ state at $1.5 \mathrm{MeV}$ which is $42 \%$ occupied. This is the only lowenergy state that can contribute to some extent to the excitation mode. The states that are completely empty and can thus contribute more to the excitation are located at higher energies. The first is an $f_{7 / 2}$ state at $5.8 \mathrm{MeV}$. The others have larger energies (at least $1 \mathrm{MeV}$ more). One can thus expect that the unperturbed response profile starts with a peak at twice $5.8 \mathrm{MeV}$, i.e. at $\sim 11.6$ $\mathrm{MeV}$ (with some small contribution at $3 \mathrm{MeV}$ ). In the case of a surface interaction, $\eta=1$, again, all the states between 0 and $5 \mathrm{MeV}$ are occupied with the exception of a $h_{11 / 2}$ state at $2.2 \mathrm{MeV}$ ( $42 \%$ of occupation). This time there are several unoccupied states just above $5 \mathrm{MeV}$, the lowest energy being at $5.4 \mathrm{MeV}\left(p_{3 / 2}\right.$ state). Hence, the unperturbed response is expected to have some structure starting from $\sim 10.8 \mathrm{MeV}$ with a small contribution at $\sim 4.4 \mathrm{MeV}$.

For the nucleus ${ }^{136} \mathrm{Sn}$ the situation is different: there are several low-lying unoccupied states. For $\eta=0.35$ the lowest energy for a completely unoccupied state is 1.9 $\mathrm{MeV}\left(p_{3 / 2}\right.$ state). At $0.8 \mathrm{MeV}$ one also finds a $f_{7 / 2}$ state with $45 \%$ of occupation. In the case $\eta=1$ the lowest energy for a totally unoccupied state is $1.7 \mathrm{MeV}\left(p_{3 / 2}\right.$ state) and a $f_{7 / 2}$ state is found at $1.6 \mathrm{MeV}$ with $32 \%$ of occupation. The unperturbed response is expected to start at $\sim 3.8$ and $3.2 \mathrm{MeV}$ for $\eta=0.35$ and 1 , respectively. In the former case a small contribution at $\sim 1.6$ $\mathrm{MeV}$ is also expected.

In order to disentangle between the various pairing effects, the unperturbed response in the two neutrons addition mode is first shown on Fig. 4 for ${ }^{124} \mathrm{Sn}$. The unperturbed response is built on the HFB single quasiparticle (QP) spectrum, for the three pairing interactions. It should be noted that the spectrum is showed above 10 $\mathrm{MeV}$, because there is only the $\mathrm{h}_{11 / 2}$ subshell which can welcome two neutrons to make a low energy state: all the other configurations belong to the next major shell (see Table II), explaining this high energy feature of the spectrum, as stated above. For all the mixed pairing interaction, the unperturbed spectrum is similar, showing that both the single quasiparticle energy and wave functions are close to each other in that case. However, in the
TABLE II: Neutron quasiparticle states with $E \leq 6 \mathrm{MeV}$ and occupation less than $80 \%$. The nucleus is ${ }^{124} \mathrm{Sn}$.

\begin{tabular}{cccc}
\hline \hline$\eta$ & State & $E(\mathrm{MeV})$ & occ \\
\hline 0.35 & $\mathrm{~h} 11 / 2$ & 1.5 & 0.42 \\
& $\mathrm{f} 7 / 2$ & 5.8 & 0.01 \\
\hline 0.65 & $\mathrm{~h} 11 / 2$ & 1.7 & 0.42 \\
& $\mathrm{f} 7 / 2$ & 5.7 & 0.01 \\
\hline 1 & $\mathrm{~h} 11 / 2$ & 2.2 & 0.42 \\
& $\mathrm{p} 3 / 2$ & 5.4 & 0.003 \\
& $\mathrm{f} 7 / 2$ & 5.5 & 0.02 \\
& $\mathrm{p} 1 / 2$ & 5.6 & 0.002 \\
& $\mathrm{~s} 1 / 2$ & 5.7 & 0.002 \\
\hline \hline
\end{tabular}

TABLE III: Same as in Table II but for ${ }^{136} \mathrm{Sn}$.

\begin{tabular}{|c|c|c|c|}
\hline$\eta$ & State & $E(\mathrm{MeV})$ & occ \\
\hline \multirow[t]{9}{*}{0.35} & $\mathrm{f} 7 / 2$ & 0.8 & 0.45 \\
\hline & $\mathrm{p} 3 / 2$ & 1.9 & 0.01 \\
\hline & $\mathrm{p} 1 / 2$ & 2.4 & 0.006 \\
\hline & $\mathrm{f} 5 / 2$ & 2.9 & 0.01 \\
\hline & $\mathrm{s} 1 / 2$ & 3.3 & 0.0005 \\
\hline & $\mathrm{d} 5 / 2$ & 4.0 & 0.0002 \\
\hline & $\mathrm{d} 3 / 2$ & 4.1 & 0.0005 \\
\hline & g9/2 & 5.6 & 0.0001 \\
\hline & $\mathrm{g} 7 / 2$ & 5.6 & 0.0001 \\
\hline \multirow[t]{9}{*}{0.65} & $\mathrm{f} 7 / 2$ & 0.9 & 0.43 \\
\hline & $\mathrm{p} 3 / 2$ & 1.9 & 0.02 \\
\hline & $\mathrm{p} 1 / 2$ & 2.4 & 0.008 \\
\hline & $\mathrm{f} 5 / 2$ & 2.9 & 0.02 \\
\hline & $\mathrm{s} 1 / 2$ & 3.2 & 0.0004 \\
\hline & $\mathrm{d} 5 / 2$ & 3.9 & 0.0001 \\
\hline & $\mathrm{d} 3 / 2$ & 3.9 & 0.0003 \\
\hline & g9/2 & 5.4 & 0.0001 \\
\hline & $\mathrm{g} 7 / 2$ & 5.5 & 0.00003 \\
\hline \multirow[t]{9}{*}{1} & $\mathrm{f} 7 / 2$ & 1.6 & 0.32 \\
\hline & $\mathrm{p} 3 / 2$ & 1.7 & 0.02 \\
\hline & $\mathrm{p} 1 / 2$ & 1.9 & 0.01 \\
\hline & $\mathrm{s} 1 / 2$ & 1.9 & 0.0004 \\
\hline & $\mathrm{d} 5 / 2$ & 2.6 & 0.0003 \\
\hline & $\mathrm{d} 3 / 2$ & 2.6 & 0.0002 \\
\hline & $\mathrm{f} 5 / 2$ & 3.0 & 0.01 \\
\hline & g9/2 & 4.1 & 0.0002 \\
\hline & $\mathrm{g} 7 / 2$ & 4.1 & 0.0001 \\
\hline
\end{tabular}

case of the pure surface pairing, the spectrum is changed. The energies are shifted to lower values, and the overall strength is increased. The lower energy shift can be understood by more single QP states located at low energy. This can be explained by a lower pairing gap and a different energy spectrum found in the HFB self-consistent 


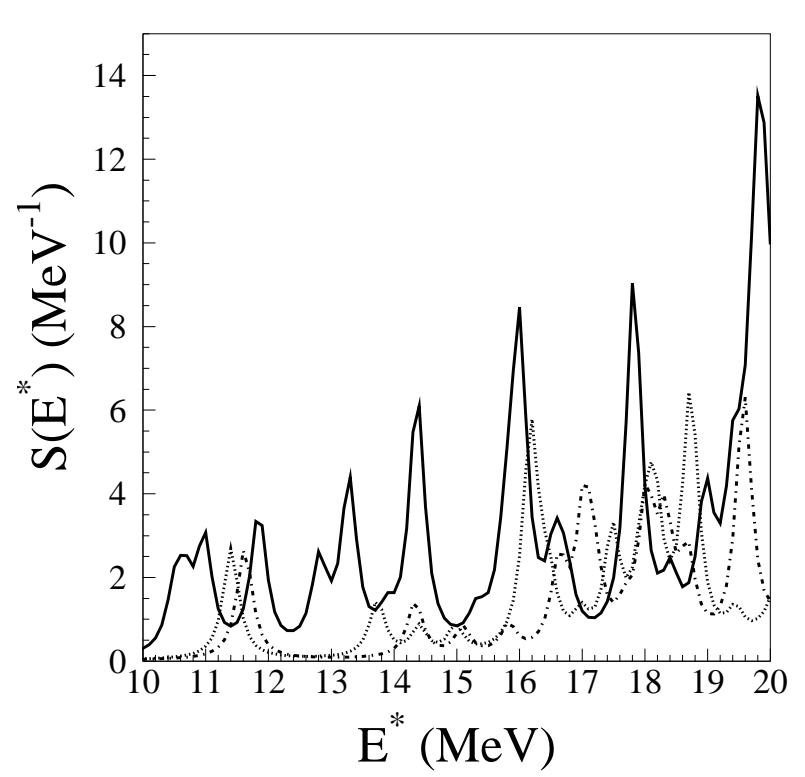

FIG. 4: Unperturbed response function for ${ }^{124} \mathrm{Sn}$ in the two neutrons $0^{+}$addition mode. The pure surface mode is in solid line, the $\eta=0.65$ mode is in dotted line, and the $\eta=0.35$ mode in dashed-dotted lines

procedure. The larger magnitude comes from the wave functions, and will be studied with the QPRA response. It can be already stated that the QRPA response will also have more strength at lower energy, due to this peculiar feature of the unperturbed spectrum for the pure surface pairing force.

Fig. 5 shows the unperturbed response for the two neutron addition mode in ${ }^{136} \mathrm{Sn}$. In this case, at the beginning of an open neutron shell several low energies configurations can welcome the two neutrons (see Table III). As in the case of ${ }^{124} \mathrm{Sn}$, the response exhibits larger strength at low energy in the specific case of the pure surface pairing interaction, compared to others pairing interaction. This is related to the pairing field profile as shown on Fig. 1. It should be noted that in order to clearly see the effect due to the surface pairing, not only the first $0^{+}$state, but also the energy area of a few $\mathrm{MeV}$ above should be explored since the results are different from 0 to $4 \mathrm{MeV}$ on Fig. 5 .

\section{Perturbed response results}

Fig. 6 shows the QRPA response for ${ }^{124} \mathrm{Sn}$, with a pure surface and the two mixed interactions. As expected the residual interaction plays a similar role in all the cases, gathering strength and shifting it to lower energy. In the case of ${ }^{124} \mathrm{Sn}$, a peak around $9 \mathrm{MeV}$ is the strongest for the surface pairing interaction, to be compared with the one around $10 \mathrm{MeV}$ for the other interactions. Hence it

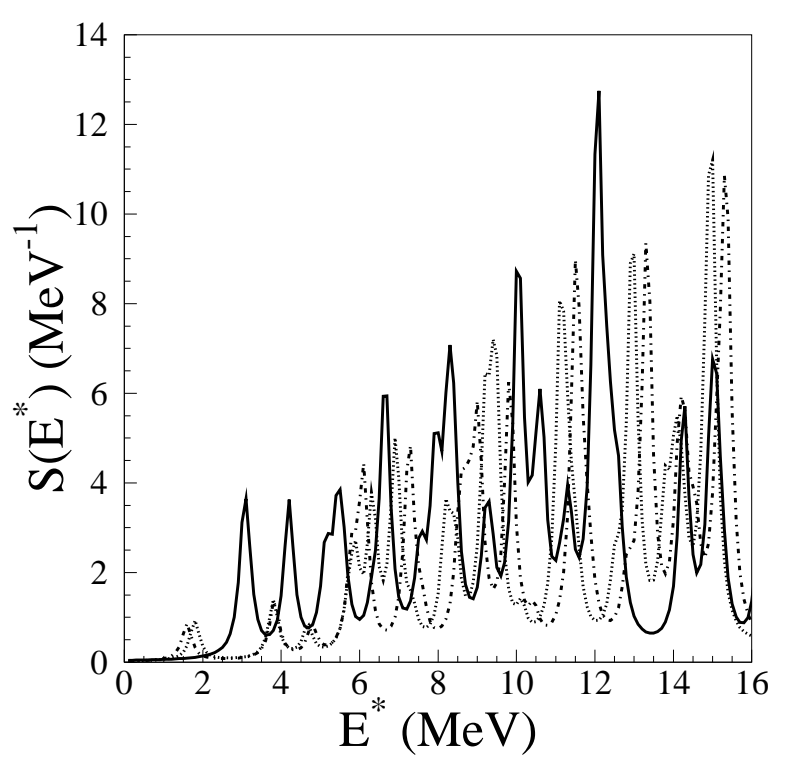

FIG. 5: Unperturbed response function for ${ }^{136} \mathrm{Sn}$ in the two neutrons $0^{+}$addition mode. The pure surface mode is in solid line, the $\eta=0.65$ mode is in dotted line, and the $\eta=0.35$ mode in dashed-dotted lines.

is expected that the pairing vibration transition strength should be larger in the case of a pure surface force. However it is known that it is difficult to describe accurately the magnitude of these transitions, especially for absolute cross section calculations 20]: one-step or sequential two-step process, triton wave function, zero-range or finite-range DWBA have to be considered. It is therefore necessary to rely on the angular distribution, calculated from the form factor, related itself to the pairing transition density 13 .

The pairing transition density is defined as:

$$
\kappa^{\nu}(\mathbf{r}, \sigma)=\langle 0|c(\mathbf{r}, \bar{\sigma}) c(\mathbf{r}, \sigma)| \nu\rangle
$$

where $c^{\dagger}(\mathbf{r}, \bar{\sigma})=-2 \sigma c^{\dagger}(\mathbf{r},-\sigma)$ is its time reversed counterpart.

It allows to calculate the form factor in the zero-range DWBA approximation. The pairing transition densities of Fig. 17 show, in the case of ${ }^{124} \mathrm{Sn}$, a difference, going from surface to other modes: the transition density decreases at the surface. However the difference is not dramatic and may be overruled by the experimental uncertainties. The larger strength of the $9 \mathrm{MeV}$ peak in the pure surface pairing interaction is due to a larger density at the surface.

For the ${ }^{136} \mathrm{Sn}$ neutron-rich nucleus, the low energy spectrum displayed on Fig. 8 is dramatically changed from using surface to other interactions, on a several $\mathrm{MeV}$ area. A three peaks structure appears in the surface case, compared to the 2 peak structure of the other cases. The integrated strength is also larger in the surface case. 


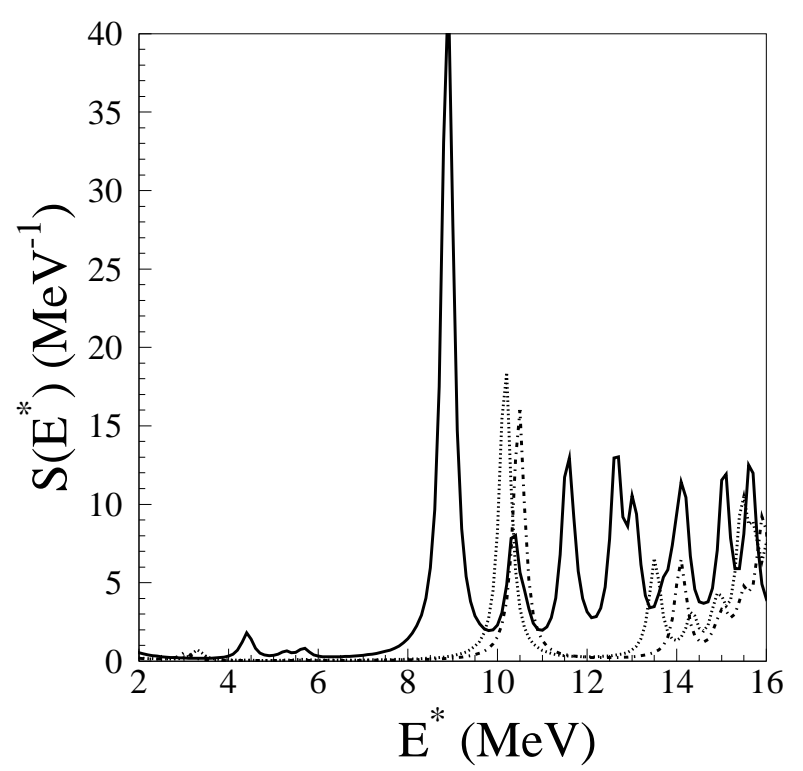

FIG. 6: QRPA response function for ${ }^{124} \mathrm{Sn}$ in the two neutrons $0^{+}$addition mode. The pure surface mode is in solid line, the $\eta=0.65$ mode is in dotted line, and the $\eta=0.35$ mode in dashed-dotted lines.

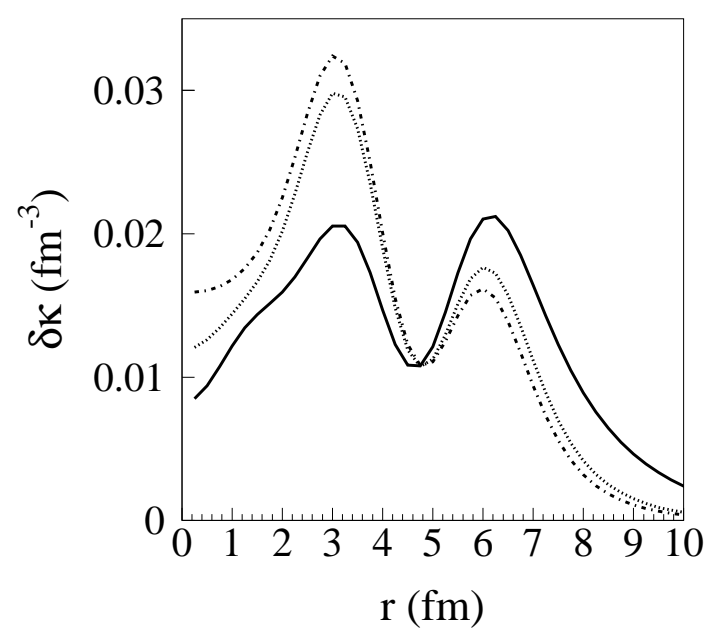

FIG. 7: Neutron transition density in the two neutrons addition mode for ${ }^{124} \mathrm{Sn}$ for the first peak located at 9-10 MeV. The pure surface mode is in solid line, the $\eta=0.65$ mode is in dotted line, and the $\eta=0.35$ mode in dashed-dotted lines.

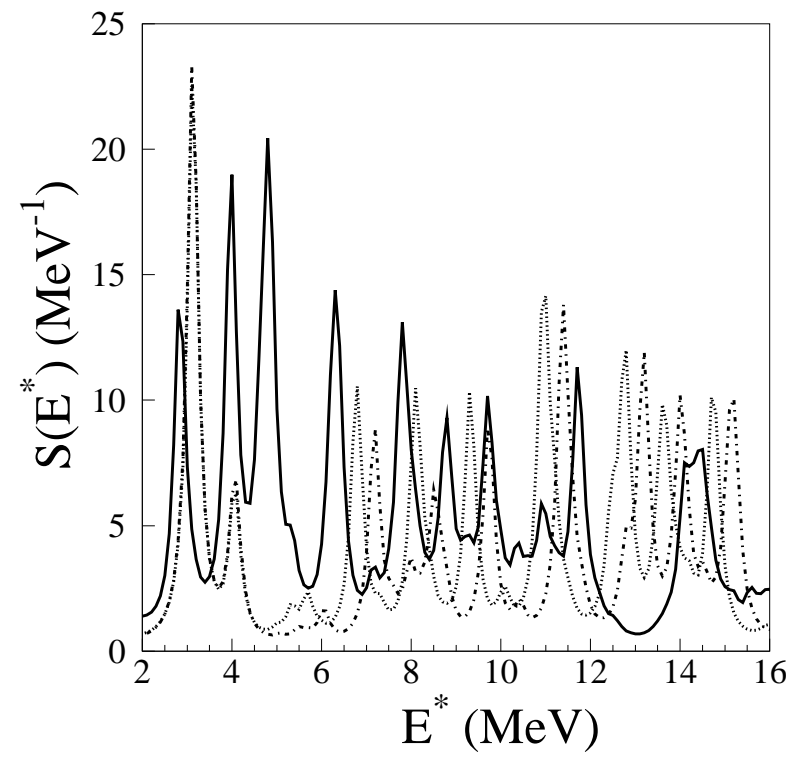

FIG. 8: QRPA response function for ${ }^{136} \mathrm{Sn}$ in the two neutrons $0^{+}$addition mode. The pure surface mode is in solid line, the $\eta=0.65$ mode is in dotted line, and the $\eta=0.35$ mode in dashed-dotted lines.

Fig. 9 and 10 show the corresponding transition densities. They exhibit very different shapes, comparing results with the pure surface pairing interaction and the mixed pairing interaction. Hence ${ }^{136} \mathrm{Sn}$ is a good test case to probe the pairing interaction through pairing vibrations. For instance in the case of the most intense peak, the central part is dominant in the transition density for the mixed case, whereas the surface part of the transition density dominates in the pure surface interaction. Hence a measurement of the angular distributions associated with the pairing vibration strength in very neutron rich-nuclei such as ${ }^{136} \mathrm{Sn}$ seems more decisive to disentangle between the pairing interactions than with ${ }^{136} \mathrm{Sn}$. This may be due to the larger neutron skin in ${ }^{136} \mathrm{Sn}$, consisting of low density neutron-rich matter.

It has been shown in a previous work how the pairing transition densities allow to calculate the two neutron form factor in order to predict angular distributions for pairing vibrations [14]. Work along these lines should be undertaken in order to bring additional constrains on the pairing interaction.

\section{CONCLUSIONS}

The impact of various pairing interactions on pairing vibrations predictions has been analysed for the first time using a HFB+QRPA approach. They should provide a good sensitivity from a pure surface interaction compared to mixed interactions, especially in the case 


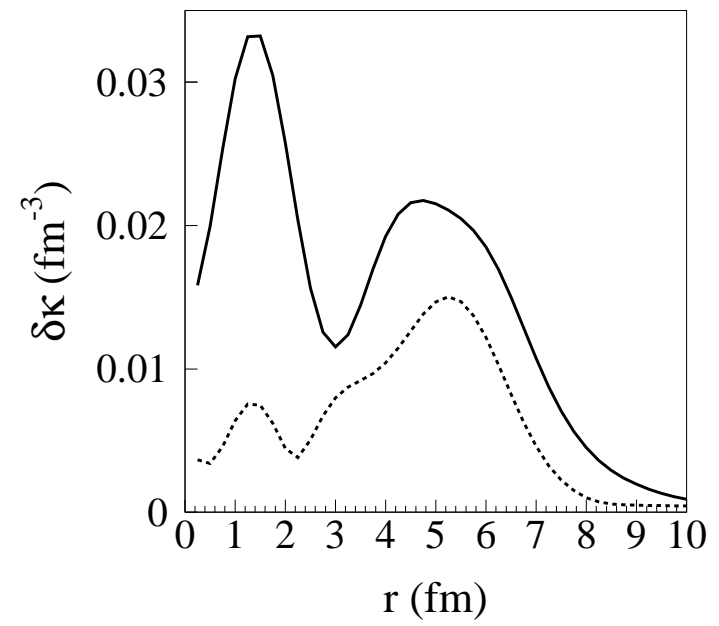

FIG. 9: Neutron transition density in the two neutrons addition mode for ${ }^{136} \mathrm{Sn}$ for the first two peaks of the strength, in the case of the mixed $\eta=0.65$ interaction of very neutron-rich nuclei such as ${ }^{136} \mathrm{Sn}$. Moreover nuclear matter gap calculations show that the LDA in the pairing channel is valid in the surface of the nuclei for the $\eta=0.35$ mixed surface/volume interaction, but not rigorously valid in other cases. In the case of exotic nuclei, pairing vibrations are found more sensitive to the surface/volume type of the pairing interaction, than in the case of stable nuclei. This may be due to the larger extension of the neutron density in very neutron-rich nuclei.

The same study using an isospin dependent pairing interaction will be undertaken. The hope is to come one step closer to a more global pairing interaction, using odd even mass staggering, pairing vibrations, and nuclear matter as constraints. Experimentally, the pairing transition densities can be tested through the form factor used to calculate the two neutrons transfer cross section. This implies to use a adequate reaction model. Work along these lines will be undertaken in an near future.

Acknowledgements The authors thank D. Beaumel, M. Matsuo and A. Vitturi for fruitful discussions
[1] D. J. Dean and M. Hjorth-Jensen, Rev. Mod. Phys. 75, $607(2003)$

[2] E. Garrido, P. Sarriguren, E. Moya de Guerra, P. Schuck, Phys. Rev C60 (1999) 064312.

[3] J. Dobaczewski, H. Flocard, and J. Treiner, Nucl. Phys. A 422, 103 (1984)

[4] S. Goriely, M. Samyn, and J. M. Pearson, Phys. Rev. C75, 064312 (2007)

[5] N. Pillet, N. Sandulescu, P. Schuck, Phys. Rev. C76 024310 (2007).

[6] M. Matsuo, K. Mizuyama, Y. Serizawa, Phys. Rev. C71 064326 (2005).

[7] K. Hagino and H. Sagawa, Phys. Rev. C76, 047302 (2007)

[8] M. Matsuo, Phys. Rev. C73 044309 (2006).

[9] K. Hagino, H. Sagawa, J. Carbonell, and P. Schuck Phys. Rev. Lett. 99, 022506 (2007)

[10] J. Margueron, H. Sagawa, K. Hagino, Phys. Rev. C76 064316 (2007)
[11] J. Margueron, H. Sagawa, K. Hagino, Phys. Rev. C77 054309 (2008).

[12] R. A. Broglia, O. Hansen and C. Riedel, Advances in Nuclear Physics, NY Plenum 6(1973) 287.

[13] W. von Oertzen, A. Vitturi, Rep. Prog. Phys. 64(2001) 1247.

[14] E. Khan, N. Sandulescu, Nguyen Van Giai, M. Grasso, Phys. Rev. C69 014314 (2004).

[15] B. Avez, C. Simenel, Ph. Chomaz, Phys. Rev. C78 044318 (2008)

[16] E. Chabanat, P. Bonche, P. Haensel, J. Meyer, R. Schaeffer, Nucl. Phys. A635 (1998) 231.

[17] E. Khan, N. Sandulescu, M. Grasso, N. V. Giai, Phys. Rev. C66 (2002) 024309.

[18] M. Matsuo, Proc. of COMEX 3 Conference (2009)

[19] K. Mizuyama, M. Matsuo and Y. Serizawa Phys. Rev. C79 (2009) 024313.

[20] M. Igarashi, K. Kubo and K. Yagi, Phys. Rep. 199(1991) 


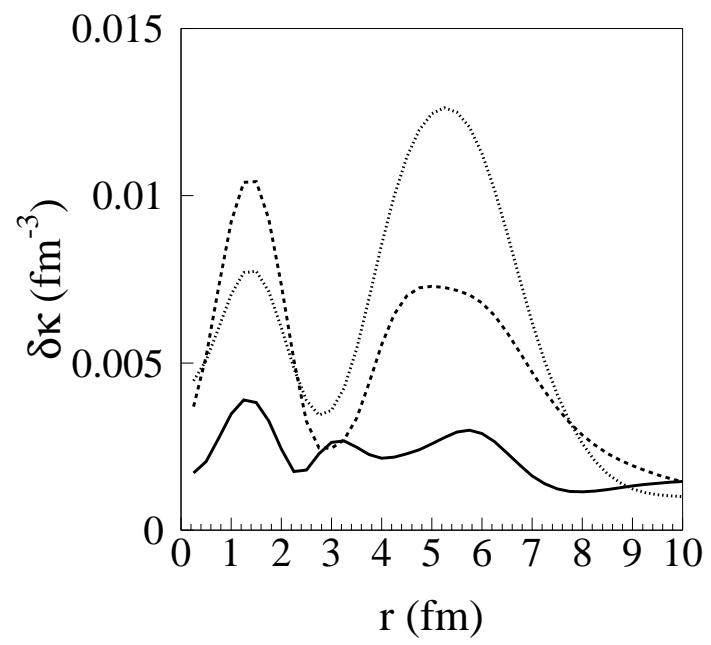

FIG. 10: Neutron transition density in the two neutrons addition mode for ${ }^{136} \mathrm{Sn}$ for the first 3 peaks of the strength, in the case of the pure surface interaction 